\title{
Observation of High Spin-to-Charge Conversion by Sputtered Bismuth Selenide Thin Films at Room Temperature Supporting Information
}

Mahendra DC ${ }^{\dagger \S}$, Jun-Yang Chen ${ }^{\ddagger}$, Thomas Peterson ${ }^{\dagger}$, Protuysh Sahu ${ }^{\dagger}$, Bin $\mathrm{Ma}^{\star}$, Naser Mousavi ${ }^{\star}$, Ramesh Harjani ${ }^{\ddagger}$, and Jian-Ping Wang ${ }^{\dagger, \ddagger *}$

${ }^{\dagger}$ School of Physics and Astronomy, University of Minnesota, MN 55455

${ }^{\ddagger}$ Department of Electrical and Computer Engineering, University of Minnesota, MN 55455

*Correspondence and requests should be addressed to Jian-Ping Wang (jpwang@umn.edu)

${ }^{\S}$ These authors contributed equally.

S1. Characterization of temperature dependent resistivity and carrier concentration of GBS thin film

S2. Intrinsic damping constant and saturation magnetization characterization of $\mathrm{Co}_{20} \mathrm{Fe}_{60} \mathrm{~B}_{20}$

S3. TEM characterization of spin-pumping sample

S4. Spin-pumping on reference Pt sample

S5. Thermal contribution in FMR spin-to-charge conversion voltage

20 S6. Fit to the figure-of-merit of spin-to-charge conversion using the

1 inverse spin Hall effect 

film.

Fig. S1(a) and (c) shows temperature dependent resistance. The resistance as a function of temperature shows that it increases with decreases in temperature, which means GBS films show semiconductor like behavior. The room-temperature resistivity of GBS $(4 \mathrm{~nm})$ is estimated to be $\sim 6000$ $\mu \Omega \mathrm{cm}$. The Hall resistance measured on Sub/ GBS (4 nm) as a function of the magnetic field is presented on Fig. S1(b). The carrier concentration obtained by linear fit on the Hall data is estimated to be $2.6 \times 10^{20}$ $\mathrm{cm}^{-3}$.
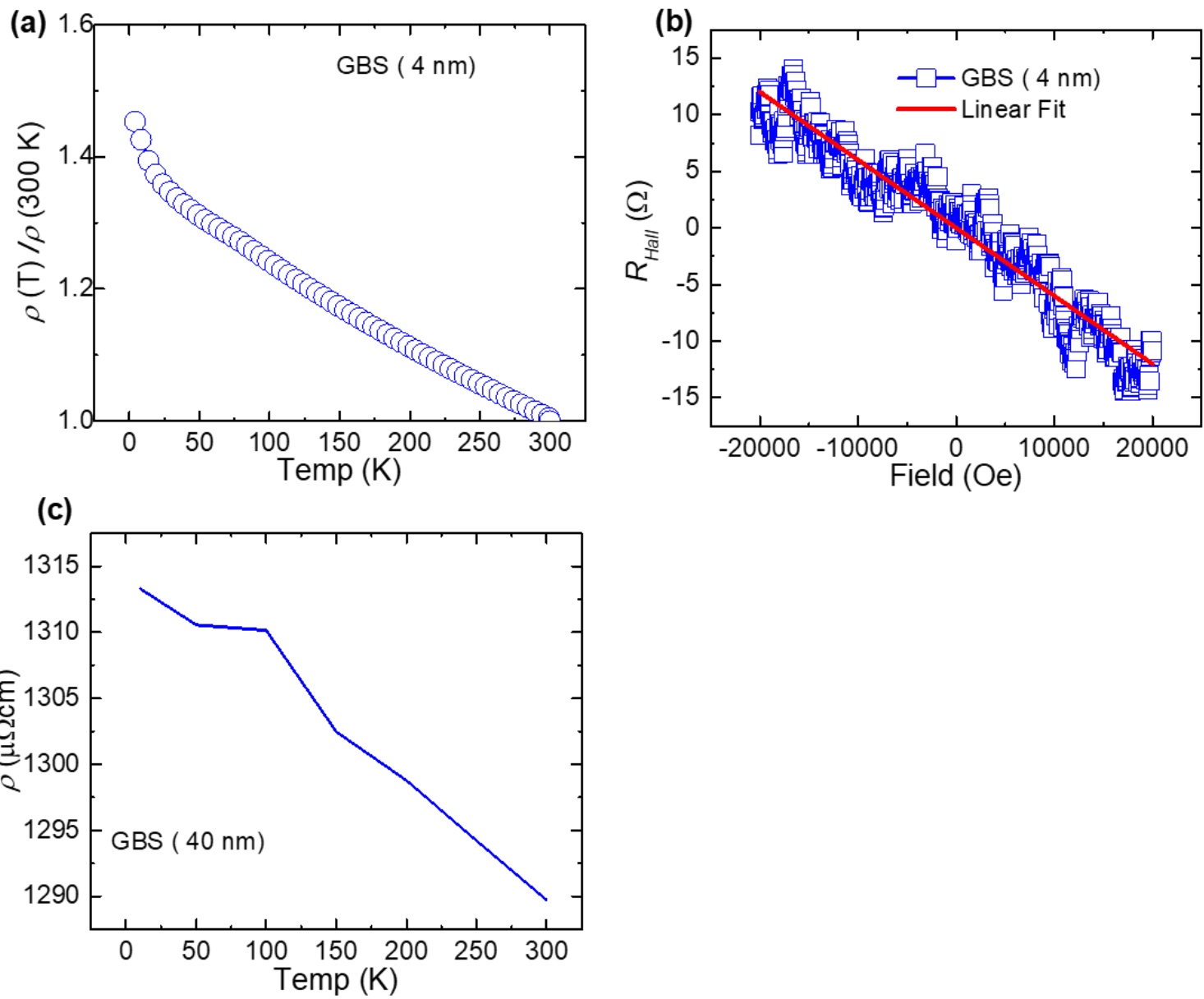
films. (a) and (c) Resistivity as a function of temperature of GBS 8 and $40 \mathrm{~nm}$ thin films, respectively. (b) Hall resistance for the characterization of carrier concentration. 


\section{S2. Intrinsic damping constant and saturation magnetization characterization of $\mathrm{Co}_{20} \mathrm{Fe}_{60} \mathrm{~B}_{20}$}

37 A control sample with the stack structure $\mathrm{Si} / \mathrm{SiO}_{2} / \mathrm{CoFeB}(5 \mathrm{~nm}) / \mathrm{MgO}(2 \mathrm{~nm}) / \mathrm{Ta}(2 \mathrm{~nm})$ was prepared to determine $\alpha$ of the CoFeB. The $V_{S P}$ of the control sample CoFeB is as shown in Fig. S2(a). As expected the $V_{A S}$ is dominant in CoFeB sample and a small $V_{S}$ is observed mainly due to the Seebeck effect and spin-orbit coupling of the $\mathrm{CoFeB}$ layer. $\Delta H$ as a function of the excitation frequency is presented in Fig. $\mathrm{S} 2$ (b). $\alpha$ determined by linear fit is 0.003 . The excitation frequency is plotted against resonance field in Fig. S2(c). The $M_{\text {eff }}$ obtained by Kittel fitting is $1.91 \times 10^{4}$ Oe.
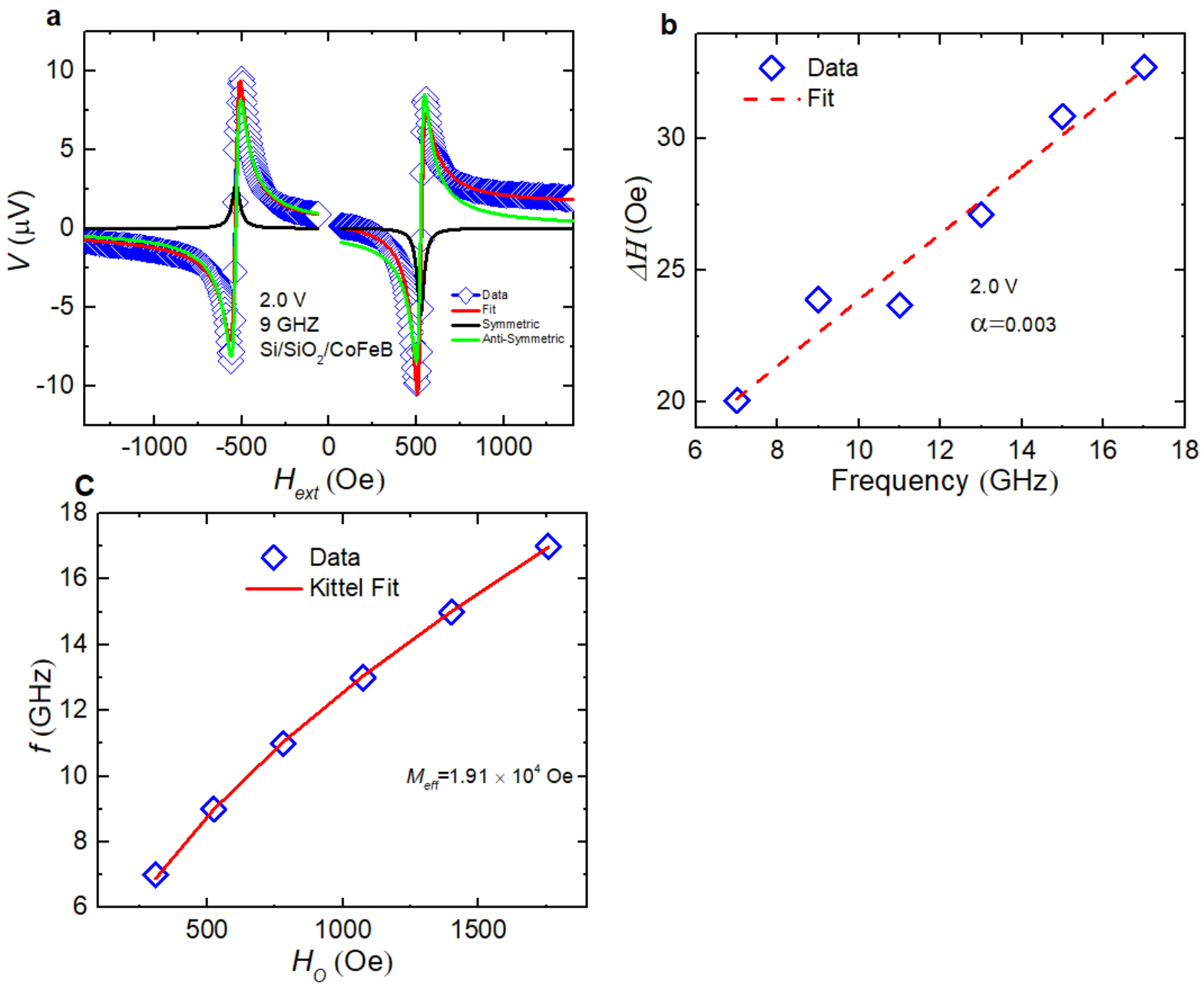

Fig. S2 Characterization of damping constant and saturation magnetization of CoFeB. (a) The spinpumping voltage measured in $\mathrm{CoFeB}$ sample at $9 \mathrm{GHz}$ excitation frequency. (b) The line-width as a function of the excitation frequency. (c) The excitation frequency as a function of resonance field at $2.0 \mathrm{~V}$ excitation amplitude. 
50

51

52

53

54

55

56

57

58

59

60

61

Bi
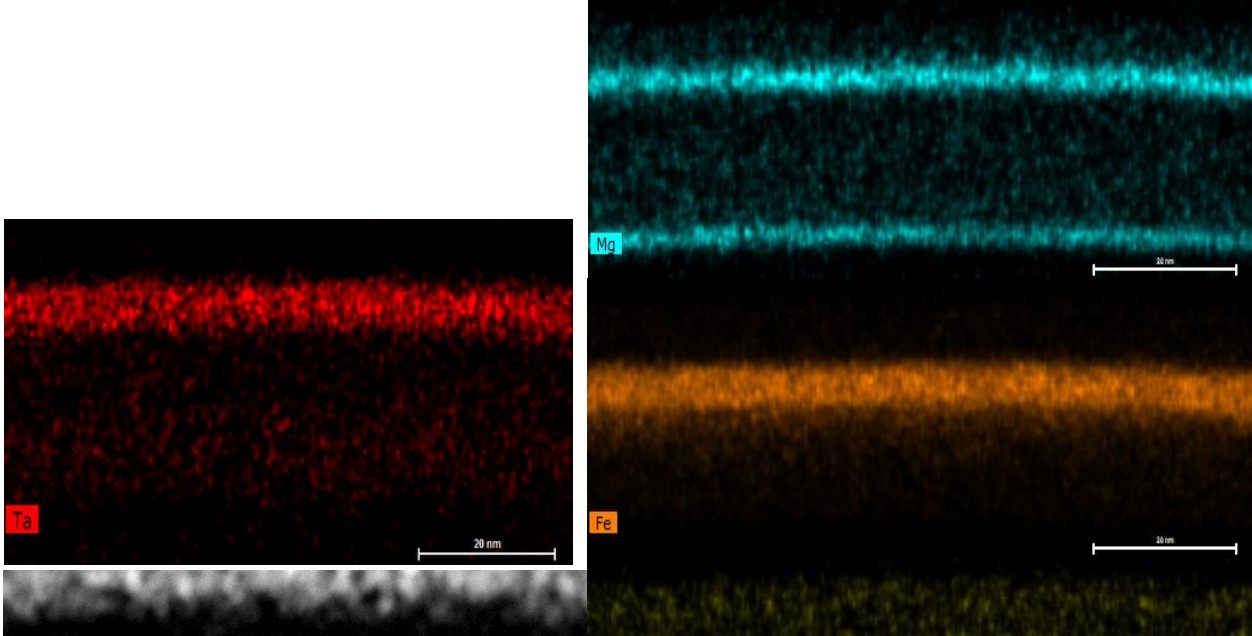

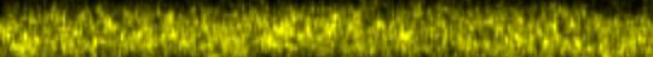

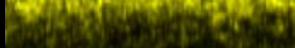

$\mathrm{Co}$

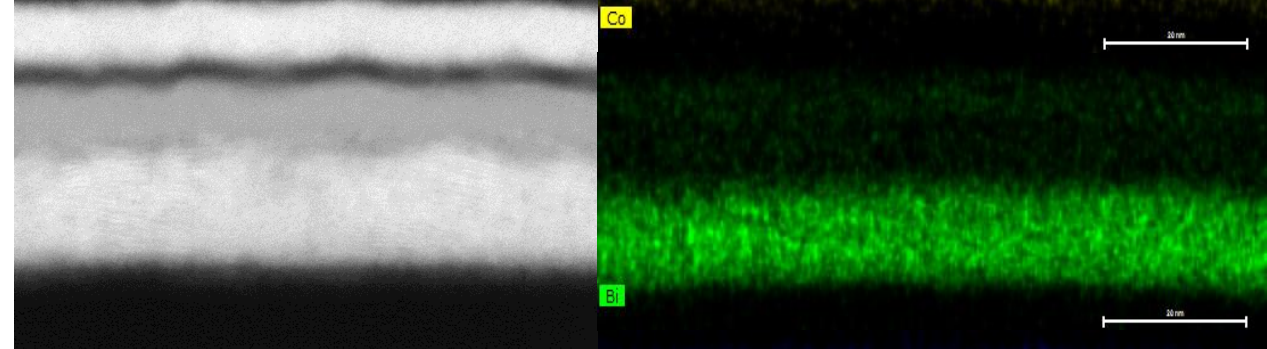

62

63

64

65

66

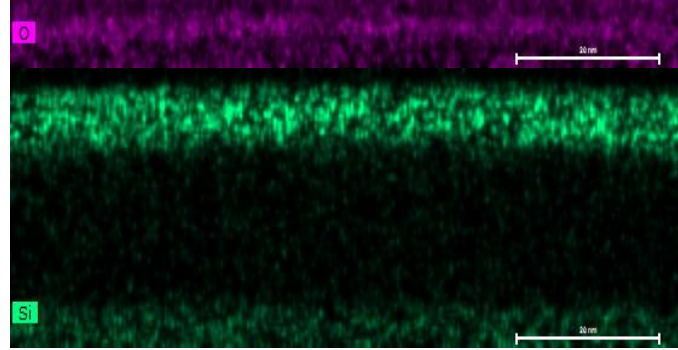

68 
Fig. S3 HAADF-STEM acquired image and individual elemental energy dispersive X-ray

70 spectroscopy (EDX) maps for BS8 sample.

\section{S4. Spin-pumping on reference Pt sample}

A reference Pt sample with stack structure $\mathrm{Si} / \mathrm{SiO}_{2} / \mathrm{Pt}(10 \mathrm{~nm}) / \mathrm{CoFeB}(5 \mathrm{~nm}) / \mathrm{MgO}(2 \mathrm{~nm}) / \mathrm{Ta}(2$

$73 \mathrm{~nm}$ ) was prepared. The $V_{S P}$ of the control sample Pt is as shown in Fig. S4(a). As expected the $V_{S}$ is

74 dominant, which corresponds to the spin-to-charge conversion voltage due to the inverse spin Hall effect

75 and a small $V_{A S}$ is due to AHE. $\Delta H$ as a function of the excitation frequency is presented in Fig. S4(b). The

76 linear fit estimated $\alpha$ is 0.0066 and $g_{\uparrow \downarrow}$ is obtained to be $1.6 \times 10^{19} \mathrm{~m}^{-2}$. The resonance frequency as a

77 function of the resonance field is presented in the Fig. S4(c). The $M_{\text {eff }}$ is estimated to be $1.76 \times 10^{4}$ Oe using

78 Kittel's formula. The $V_{\text {ISHE }}$ as a function of the excitation voltage is presented in Fig. S4(d). The estimated

$79 J_{S}$ is $5.02 \times 10^{6} \mathrm{~A} / \mathrm{cm}^{2}$ and the spin Hall angle is obtained to be 0.1 estimated by using following equation:

$80 \quad V_{I S H E}=w R \lambda \theta \tanh (x / 2 \lambda) J_{S}$

81 Where $x, \theta$ and $\lambda$ are thickness, spin Hall angle, and spin-diffusion length of the Pt, respectively. This value 82 of $\theta$ is comparable to the previous reports. 

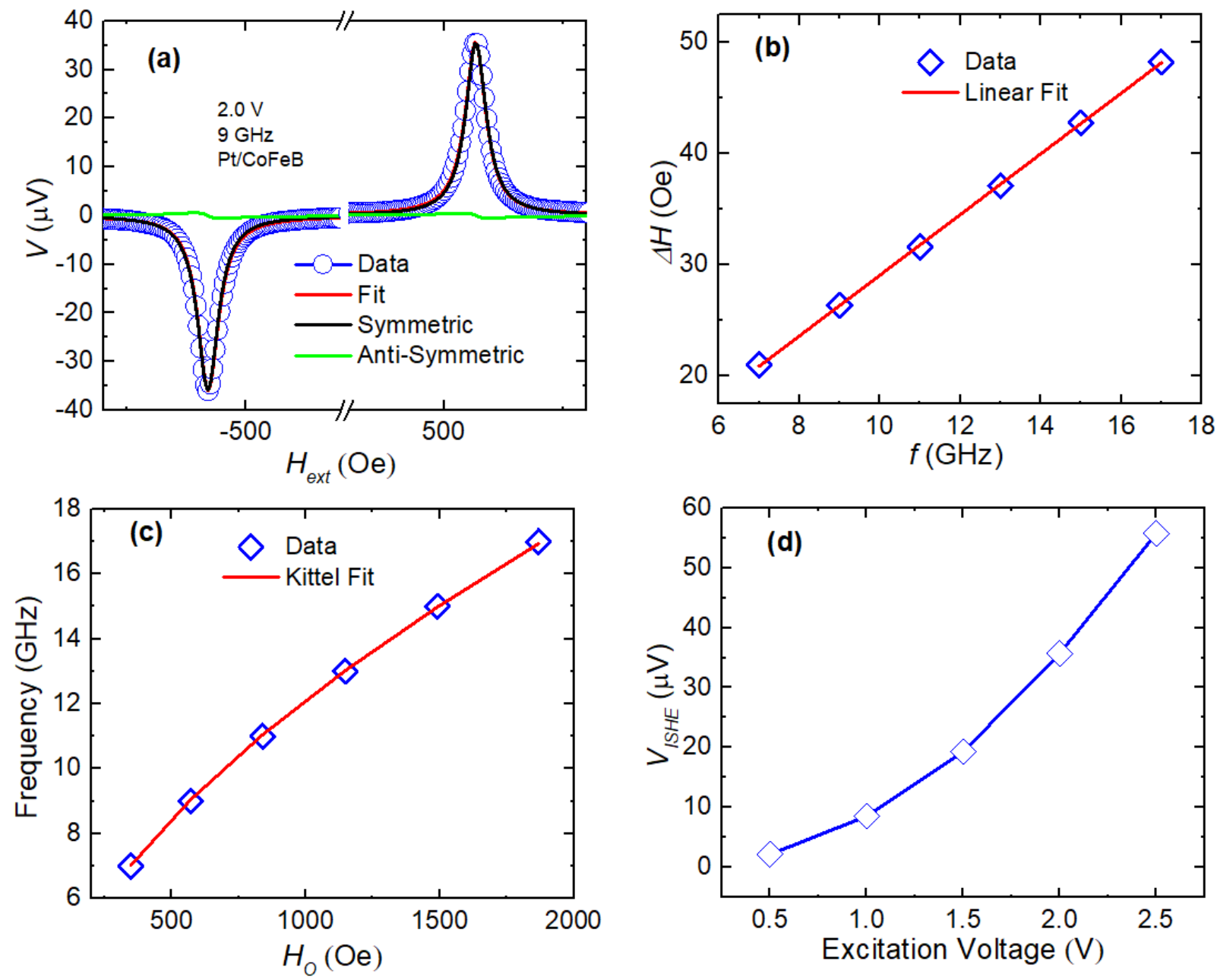

Fig. S3 Characterization of spin-to-charge conversion via ISHE in Pt/CoFeB Sample. (a) The spin-

85 pumping voltage measured in $\mathrm{Pt} / \mathrm{CoFeB}$ sample at $9 \mathrm{GHz}$ excitation frequency. (b) The line-width as a

86 function of the excitation frequency for Pt sample. (c) The excitation frequency as a function of resonance

87 at $2.0 \mathrm{~V}$ excitation amplitude. (d) The spin-pumping voltage as a function of the excitation amplitude for 88 at $9 \mathrm{GHz}$ excitation frequency. 
In FMR spin-pumping, microwave is absorbed by the ferromagnetic magnetization. The absorption of microwaves creates magnetization dynamics resulting in heat due to damping

94 process. Thus produced heat induces a temperature gradient $(\nabla T)$ both along longitudinal and vertical directions. The longitudinal $\nabla T$ is converted into an emf, $V_{S E} \propto \nabla T$, which is referred as Seebeck voltage ${ }^{1,2}$. The absorption and emission of power by magnetization is linear to the

97 intensity of microwave power $(\Delta P)$ and it is maximized at $\mathrm{FMR}^{3}$. Since microwave power

98 absorbed by magnetization is proportional to the Lorenzian $\left(\frac{\Delta H^{2}}{\Delta H^{2}+\left(H_{e x t}-H_{0}\right)^{2}}\right)^{2}$ and power absorbed is linear proportional to the thermal gradient so $V_{S E}$ will have symmetric Lorenzian line-

100 shape.

Additionally, $\nabla T$ along the perpendicular direction can drive charge carriers which under

102 the application of the static external field $\left(H_{\text {ext }}\right)$ along the y-direction can generate voltage similar

103 to ordinary Hall voltage along the $\mathrm{x}$-direction. This voltage is commonly known as the Nernst

104 Voltage $V_{N E} \propto \nabla T \times H_{e x t}{ }^{4}$. Since Bi has largest Nernst effect there could be contribution of Nernst

105 effect from our GBS ${ }^{5}$. Furthermore, $\nabla T$ along the vertical direction in the ferromagnetic film can

106 generate voltage due to the anomalous Nernst effect (ANE) given by $V_{A N E} \propto \nabla T \times \hat{m}^{6}$. Both Nernst

107 effect and ANE could couple with spin-to-charge conversion voltage due to the ISHE or IEE. If

108 Nernst and ANE contributed to spin-to-charge conversion in our GBS/CoFeB bilayer; before and

109 after the insertion of $\mathrm{MgO}$ layer the spin-to-charge conversion voltage should still be similar. 


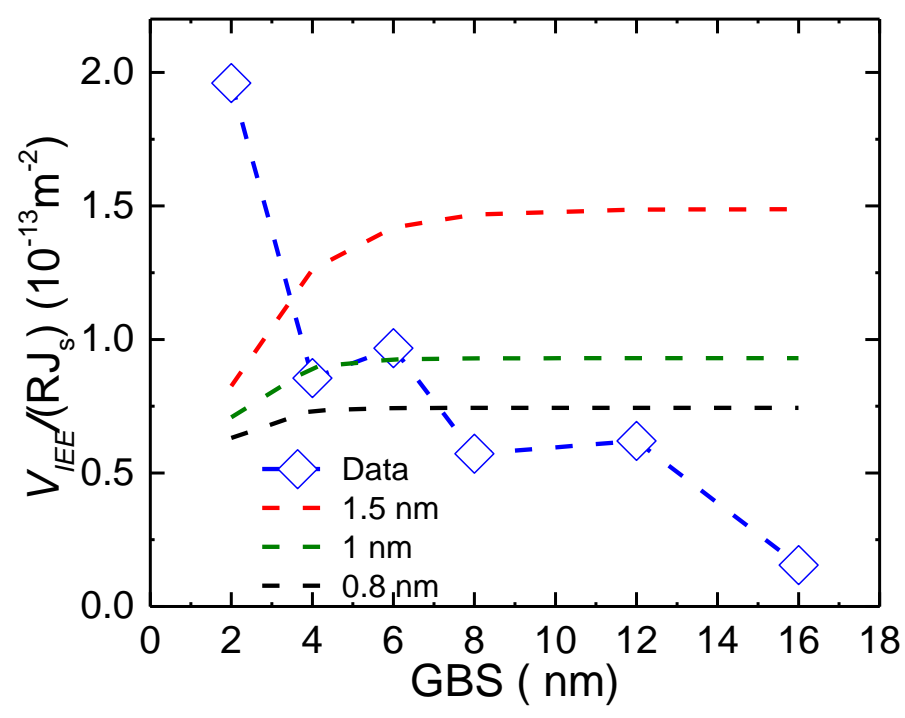

113 Fig. S5 Fit to figure-of-merit of spin-to-charge conversion versus GBS film thickness data

114 using the inverse spin Hall effect. Dashed red, green, and black lines are ISHE (eqn. S1) fit to

115 the figure-of-merit of spin-to-charge conversion data with 1.5, 1, $0.8 \mathrm{~nm}$ spin-diffusion lengths, 116 respectively.

\section{Supplementary Information References}

(1) Shiomi, Y.; Nomura, K.; Kajiwara, Y.; Eto, K.; Novak, M.; Segawa, K.; Ando, Y.; Saitoh, E. Spin-Electricity Conversion Induced by Spin Injection into Topological Insulators. Phys. Rev. Lett. 2014, 113, 196601.

(2) Iguchi, R.; Saitoh, E. Measurement of Spin Pumping Voltage Separated from Extrinsic Microwave Effects. J. Phys. Soceity Japan 2017, 86, 011003.

(3) Qiu, Z.; Uruichi, M.; Hou, D.; Uchida, K.; Yamamoto, H. M.; Saitoh, E. Spin-Current Injection

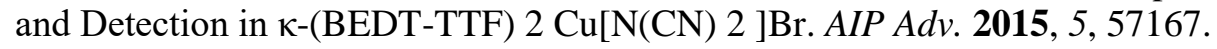

(4) Hull M Osgood, R. R.; Parisi H Warlimont, J. J.; Julian Goldsmid, H. Introduction to Thermoelectricity. Springer Ser. Mater. Sci. 2016. 
129

130

131

132

133

134

135

136

137
(5) Behnia, K.; Aubin, H. Nernst Effect in Metals and Superconductors: A Review of Concepts and Experiments Related Content Nernst Effect in Metals and Superconductors: A Review of Concepts and Experiments Review. Rep. Prog. Phys 2018, 79, 23.

(6) Avci, C. O.; Garello, K.; Gabureac, M.; Ghosh, A.; Fuhrer, A.; Alvarado, S. F.; Gambardella, P. Interplay of Spin-Orbit Torque and Thermoelectric Effects in Ferromagnet/Normal-Metal Bilayers. Phys. Rev. B 2014, 90, 224427. 